$\underline{\text { Snippets }}$

\title{
Snippets from the web
}

Sri Lanka Journal of Child Health, 2003; 32: 109-10

Statin Reverses Carotid Atherogenesis, Exercise Improves Vascular Function in Children

Two independent studies demonstrate the importance of early preventive intervention in children at risk of heart disease.

http://mp.medscape.com/cgibin1/DM/v/eeREOEIZ100DzQOFk4POAJ

\section{Routine Morphine Use Not Justified for Newborns on Ventilator Support}

In a randomized trial, there was no measurable analgesic effect or benefit on poor neurologic outcome.

http://mp.medscape.com/cgi-

bin1/DM/v/eeREOEIZ1OODzQOFk4QOAK

Flunisolide HFA Does Not Impair Growth in Children With Mild Asthma

In a study lasting 1 year, flunisolide hydrofluoroalkane (HFA), a new inhaled corticosteroid formulation, had no effect on height or growth velocity in pre-adolescents with mild asthma, according to a presentation by Dr. Leon S. Greos to the American College of Allergy, Asthma and Immunology.

http://mp.medscape.com/cgibin1/DM/v/eeREOEIZ1OODzQOFk4ROAL

\section{Children With Mild Asthma Constitute Most} Hospital Admissions for Asthma

Mild asthma accounts for the majority of hospital admissions among children with asthma, according to findings presented here on Saturday during the Annual Meeting of the American College of Allergy, Asthma, and Immunology.

http://mp.medscape.com/cgibin1/DM/v/eeREOEIZ1OODzQOFk4UOAO 
Oral Immunoglobulin Promising in Refractory Juvenile Arthritis

Results of a phase II study suggest that oral immunoglobulin (IgG) may have a role as add-on therapy for refractory juvenile rheumatoid arthritis (JRA).

http://mp.medscape.com/cgi-

bin1/DM/v/eeFfOEIZ1OODzQOFkAHOAi

\section{Child Blindness More Common Than Expected}

Childhood blindness is Britain is more common than previously thought, doctors report in the October 25th issue of The Lancet, and youngsters from ethnic minorities and lower economic groups and premature infants are vulnerable.

http://mp.medscape.com/cai-binl /DM/v/eeFfOEIZ1 OODzQOFkAMOAo

\section{Receptor Gene Plays Key Role in Regulating Puberty}

An intact $G$ protein-coupled receptor gene (GPR54) seems to be necessary for normal puberty to occur in both humans and mice, according to a report published in the October 23rd issue of The New England Journal of Medicine.

http://mp.medscape.com/cgibin1/DM/v/eeFfOEIZ100DzQOFkANOAp

Stereotactic Radiotherapy Effective for Low-Grade Gliomas in Children

In a prospective trial, survival rates were $98 \%$ at five years and $82 \%$ after eight years.

http://mp.medscape.com/cgibin1/DM/v/ed830EIZ1OODzQOFiooOA2
Low-Dose Theophylline Highly Effective for Asthma Control

But the drug is antagonistic when taken with corticosteroids, resulting in a worsening of lung function.

http://mp.medscape.com/caibin1/DM/v/eeFfOEIZ100DzQOFkAJOAI

Topical Povidone-lodine Effective in Chronic Suppurative Otitis Media

In a double-blind, randomized trial, this treatment was as effective as topical ciprofloxacin, with added benefits of reduced cost of therapy and no in vitro drug resistance.

http://mp.medscape.com/cgipin1/DM/v/eeFfOEIZ1OODzQOFkALOAn

Creatine Supplements May Improve Muscle Strength in Young Children With DMD

Children with Duchenne muscular dystrophy between the ages of 5 and 7 years show a slowing in disease progression with creatine.

http://mp.medscape.com/cgibinl/DM/v/ed830EIZ1 OODzQOFiofOAs

B J C Perera Joint Editor 


\section{Snippets from the world wide web}

Sri Lanka Journal of Child Health, 2007; 36: 138-9

\section{Reduced-Dose Hepatitis A Vaccine Effective in Children}

A paediatric formulation of the virosomebased hepatitis A virus (HAV) vaccine Epaxal (Berna Biotech) appears to be protective in children, according to European researchers.

http://mp.medscape.com/cgibin1/DM/y/eBYcn0EIZ1O0DzQ0Ix510Ey

\section{S. pneumoniae Genotype Seen Resistant to} All FDA-Approved Antibiotics

A number of Streptococcus pneumoniae strains that cause acute otitis media and invasive disease in children have been identified that are not included in the 7-valent conjugate pneumococcal vaccine. One strain in particular, expressing a serotype 19A capsule belonging to a new genotype, is highly virulent and resistant to all antibiotics that are currently approved for use in children by the US Food and Drug Administration, according to physicians in New York who identified this otopathogen.

http://mp.medscape.com/cgi-

bin1/DM/y/eBYcn0EIZ1O0DzQ0Ix7e0Et

Repeat Dosing of Antenatal Corticosteroids May Not Harm Infants at Risk for Preterm Birth

Two studies show that repeat dosing of antenatal corticosteroids in infants at risk for preterm birth is not associated with adverse effects at ages 2 to 3 years, but further study is warranted.

http://mp.medscape.com/cgibin1/DM/y/eBYcn0EIZ1O0DzQ0Ix7Z0Ei

\section{Short-Course Isoniazid Plus Rifampin Effective for Latent TB in Children}

A study shows a 3- or 4-month regimen of isoniazid plus rifampin is as effective as a 9month course of isoniazid monotherapy in treating latent tuberculosis in children.

http://mp.medscape.com/cgibin1/DM/y/eBYcn0EIZ1O0DzQ0Ix7a0Ep
Treatment Interruption in Gaucher Disease Can Cause Irreversible Complications

Interruption of enzyme replacement therapy in children with type 1 Gaucher disease can cause recurrent organomegaly, growth delays, and skeletal manifestations that do not resolve after reinstatement of treatment, according to a study published in the August issue of the Journal of Paediatrics.

http://mp.medscape.com/cgibin1/DM/y/eBYcn0EIZ1O0DzQ0IyDr0EM

Tomatoes, Cucumbers, and Fish Protect Against Childhood Wheeze and Atopy

A study shows that a diet rich in fish and fruity vegetables, such as tomatoes and cucumbers, has a protective effect against childhood wheeze and atopy,

http://mp.medscape.com/cgibin1/DM/y/eBYcn0EIZ1O0DzQ0Ix5n0E1

Cystinosin Gene May Be Tied to Cognitive Deficits

Defects in visual processing observed in young children with cystinosis indicate that the cystinosin gene may be involved, Californiabased researchers report in the Journal of Paediatrics.

http://mp.medscape.com/cgibin1/DM/y/eBYcn0EIZ1O0DzQ0IyBr0EK

Breast-Feeding May Not Reduce Risk for Asthma or Allergy

In a large cluster randomized study, prolonged or exclusive breast-feeding did not reduce the risk for asthma, hay fever, or eczema at the age of 6.5 years.

http://mp.medscape.com/cgibin1/DM/y/eBYcn0EIZ1O0DzQ0Ix5w0EB 
Children With Amblyopia May Not Need Occlusion Prescribed for More Than 6 Hours Daily

Substantial (6 hours/day) and maximal (12 hours/day) prescribed occlusion resulted in similar visual outcome, but actual use of occlusion in both groups was much less than prescribed.

http://mp.medscape.com/cgibin1/DM/y/eBYcn0EIZ1O0DzQ0Ix7Q0EZ

Older Age at Diagnosis a Risk Factor for Mortality in Childhood-Onset Diabetes

The risk of dying among patients with childhood-onset diabetes is highest for those diagnosed "at the threshold of adulthood," according to findings published in the October issue of Diabetes Care.

http://mp.medscape.com/cgibin1/DM/y/eBcL70EIZ1O0DzQ0I5nu0Ep

Use of Opiates in Children With Suspected Appendicitis Not Contraindicated

In a randomized, double-blind, placebocontrolled trial, use of opiates in children with abdominal pain suggesting appendicitis did not delay the surgical decision.

http://mp.medscape.com/cgibin1/DM/y/eBbkI0EIZ1O0DzQ0I4yC0Ex

Nitric Oxide May Help Screen for Asthma in Young Adults

The fraction of exhaled nitric oxide measurement with a portable analyzer was an effective tool to screen for asthma in young adults.

http://mp.medscape.com/cgibin1/DM/y/eBbkI0EIZ1O0DzQ0I4xb0ET

Few Diabetic Children With Infusion Pumps Achieve Optimal $\mathrm{Hg}$ AlC

Only a minority of children with type I diabetes reach their target level of glycated hemoglobin, even when using "the best insulin delivery system currently available," according to a study conducted at the University of Wisconsin School of Medicine in Madison.

http://mp.medscape.com/cgibin1/DM/y/eBbkI0EIZ1O0DzQ0I4yj0Ec
AMP May Be Better Than Methacholine in Pediatric Asthma Assessment

Bronchial hyper-responsiveness (BHR) to challenge with adenosine 5'-monophosphate (AMP) may yield more informative results in young children than does response to methacholine challenge, according to Korean researchers.

http://mp.medscape.com/cgibin1/DM/y/eBbkI0EIZ1O0DzQ0I4xh0EZ

Adenotonsillectomy Improves Sleep, Behavior in Children With Sleep-Disordered Breathing

This study also suggests that the Paediatric Sleep Questionnaire and Conners' Parent Rating Scale-Revised Short Form are useful for screening and observing children after this procedure.

http://mp.medscape.com/cgibin1/DM/y/eBbCw0EIZ1O0DzQ0I32P0E5

Overweight Children at Increased Risk for Asthma Hospitalization

Among children who presented to the emergency department for asthma, overweight children with asthma were significantly more likely to be older and live in an impoverished area.

http://mp.medscape.com/cgibin1/DM/y/eBaiA0EIZ1O0DzQ0I3TP0EN

B J C Perera

Joint Editor 



\section{Snippets from the world wide web}

Sri Lanka Journal of Child Health, 2007; 36: 138-9

\section{Reduced-Dose Hepatitis A Vaccine Effective in Children}

A paediatric formulation of the virosomebased hepatitis A virus (HAV) vaccine Epaxal (Berna Biotech) appears to be protective in children, according to European researchers.

http://mp.medscape.com/cgi-

bin1/DM/y/eBYcn0EIZ1O0DzQ0Ix510Ey

\section{S. pneumoniae Genotype Seen Resistant to} All FDA-Approved Antibiotics

A number of Streptococcus pneumoniae strains that cause acute otitis media and invasive disease in children have been identified that are not included in the 7-valent conjugate pneumococcal vaccine. One strain in particular, expressing a serotype 19A capsule belonging to a new genotype, is highly virulent and resistant to all antibiotics that are currently approved for use in children by the US Food and Drug Administration, according to physicians in New York who identified this otopathogen.

http://mp.medscape.com/cgi-

bin1/DM/y/eBYcn0EIZ1O0DzQ0Ix7e0Et

Repeat Dosing of Antenatal Corticosteroids May Not Harm Infants at Risk for Preterm Birth

Two studies show that repeat dosing of antenatal corticosteroids in infants at risk for preterm birth is not associated with adverse effects at ages 2 to 3 years, but further study is warranted.

http://mp.medscape.com/cgi-

bin1/DM/y/eBYcn0EIZ1O0DzQ0Ix7Z0Ei

\section{Short-Course Isoniazid Plus Rifampin Effective for Latent TB in Children}

A study shows a 3- or 4-month regimen of isoniazid plus rifampin is as effective as a 9month course of isoniazid monotherapy in treating latent tuberculosis in children.

http://mp.medscape.com/cgi-

bin1/DM/y/eBYcn0EIZ1O0DzQ0Ix7a0Ep
Treatment Interruption in Gaucher Disease Can Cause Irreversible Complications

Interruption of enzyme replacement therapy in children with type 1 Gaucher disease can cause recurrent organomegaly, growth delays, and skeletal manifestations that do not resolve after reinstatement of treatment, according to a study published in the August issue of the Journal of Paediatrics.

http://mp.medscape.com/cgibin1/DM/y/eBYcn0EIZ1O0DzQ0IyDr0EM

Tomatoes, Cucumbers, and Fish Protect Against Childhood Wheeze and Atopy

A study shows that a diet rich in fish and fruity vegetables, such as tomatoes and cucumbers, has a protective effect against childhood wheeze and atopy,

http://mp.medscape.com/cgibin1/DM/y/eBYcn0EIZ1O0DzQ0Ix5n0E1

Cystinosin Gene May Be Tied to Cognitive Deficits

Defects in visual processing observed in young children with cystinosis indicate that the cystinosin gene may be involved, Californiabased researchers report in the Journal of Paediatrics.

http://mp.medscape.com/cgibin1/DM/y/eBYcn0EIZ1O0DzQ0IyBr0EK

Breast-Feeding May Not Reduce Risk for Asthma or Allergy

In a large cluster randomized study, prolonged or exclusive breast-feeding did not reduce the risk for asthma, hay fever, or eczema at the age of 6.5 years.

http://mp.medscape.com/cgibin1/DM/y/eBYcn0EIZ1O0DzQ0Ix5w0EB 
Children With Amblyopia May Not Need Occlusion Prescribed for More Than 6 Hours Daily

Substantial (6 hours/day) and maximal (12 hours/day) prescribed occlusion resulted in similar visual outcome, but actual use of occlusion in both groups was much less than prescribed.

http://mp.medscape.com/cgi-

bin1/DM/y/eBYcn0EIZ1O0DzQ0Ix7Q0EZ

Older Age at Diagnosis a Risk Factor for Mortality in Childhood-Onset Diabetes

The risk of dying among patients with childhood-onset diabetes is highest for those diagnosed "at the threshold of adulthood," according to findings published in the October issue of Diabetes Care.

http://mp.medscape.com/cgi-

bin1/DM/y/eBcL70EIZ1O0DzQ0I5nu0Ep

Use of Opiates in Children With Suspected Appendicitis Not Contraindicated

In a randomized, double-blind, placebocontrolled trial, use of opiates in children with abdominal pain suggesting appendicitis did not delay the surgical decision.

http://mp.medscape.com/cgibin1/DM/y/eBbkI0EIZ1O0DzQ0I4yC0Ex

Nitric Oxide May Help Screen for Asthma in Young Adults

The fraction of exhaled nitric oxide measurement with a portable analyzer was an effective tool to screen for asthma in young adults.

http://mp.medscape.com/cgibin1/DM/y/eBbkI0EIZ1O0DzQ0I4xb0ET

Few Diabetic Children With Infusion Pumps Achieve Optimal $\mathrm{Hg}$ AlC

Only a minority of children with type I diabetes reach their target level of glycated hemoglobin, even when using "the best insulin delivery system currently available," according to a study conducted at the University of Wisconsin School of Medicine in Madison. http://mp.medscape.com/cgibin1/DM/y/eBbkI0EIZ1O0DzQ0I4yj0Ec
AMP May Be Better Than Methacholine in Pediatric Asthma Assessment

Bronchial hyper-responsiveness (BHR) to challenge with adenosine 5'-monophosphate (AMP) may yield more informative results in young children than does response to methacholine challenge, according to Korean researchers.

http://mp.medscape.com/cgi-

bin 1/DM/y/eBbkI0EIZ1O0DzQ0I4xh0EZ

Adenotonsillectomy Improves Sleep, Behavior in Children With Sleep-Disordered Breathing

This study also suggests that the Paediatric Sleep Questionnaire and Conners' Parent Rating Scale-Revised Short Form are useful for screening and observing children after this procedure.

http://mp.medscape.com/cgibin1/DM/y/eBbCw0EIZ1O0DzQ0I32P0E5

Overweight Children at Increased Risk for Asthma Hospitalization

Among children who presented to the emergency department for asthma, overweight children with asthma were significantly more likely to be older and live in an impoverished area.

http://mp.medscape.com/cgibin1/DM/y/eBaiA0EIZ1O0DzQ0I3TP0EN

B J C Perera

Joint Edit 
